\title{
Phase diagram structures in a periodic one-dimensional exclusion process
}

\author{
Rui Jiang,,${ }^{1 *}$ Yu-Qing Wang, ${ }^{1}$ Anatoly B Kolomeisky, ${ }^{2}$ Wei Huang, ${ }^{1}$ Mao-Bin Hu, ${ }^{1}$ and Qing-Song Wu ${ }^{1}$ \\ ${ }^{1}$ State Key Laboratory of Fire Science and School of Engineering Science, University of Science and Technology of China, \\ Hefei 230026, China \\ ${ }^{2}$ Department of Chemistry, Rice University, Houston, Texas 77005-1892, USA
}

(Received 17 April 2012; revised manuscript received 18 November 2012; published 8 January 2013)

\begin{abstract}
This paper studies a periodic one-dimensional exclusion process composed of a driven part and a biased diffusive part in a mesoscopic limit. It is shown that, depending on the biased diffusion parameter $\delta$, rich phase diagram structures appear in which diverse phases have been exhibited and the density profile in the diffusive part is qualitatively different. This is because the domain wall is behaving differently. Our analytical results are in good agreement with Monte Carlo simulations.
\end{abstract}

DOI: 10.1103/PhysRevE.87.012107

PACS number(s): 64.60.Ht, 05.40.-a, 05.60.-k, 05.70.Ln

\section{INTRODUCTION}

In past years, driven diffusive systems have attracted the interest of many researchers because of the systems' critical role in understanding various nonequilibrium phenomena in chemistry, physics, and biology [1-8]. In particular, in the steady state, driven diffusive systems exhibit nonvanishing current under periodic boundary conditions, and boundary induced phase transition under open boundary conditions, which have not been observed in the counterpart of equilibrium systems [9-18].

However, the nature of these phenomena depends on whether the system is diffusive or is driven due to the presence of an external field (or self-driven [19,20] due to an internal self-produced driving force). Motivated by this fact, Hinsch and Frey [21,22] presented a model that combines the symmetric and the totally asymmetric simple exclusion process (SEP [23-26] and TASEP [1-7], respectively), which are two paradigms for diffusive and driven transport in one dimension. The model might be used to describe traffic of molecular motors in closed compartments and colloidal motion in optical traps. It is shown that in a mesoscopic limit where both dynamics compete, bulk-driven phase transitions have been identified. Inspired by the work of Hinsch and Frey, Shi et al. [27] studied a periodic driven diffusive system, which separates into two equal-sized parts with different values of hopping rates. Competition of the two different driven parts leads to various bulk-driven phase transitions, including shock and antishock. More interestingly, for the symmetric scenario, one can observe shock and antishock simultaneously in the system.

This paper generalizes the work of Hinsch and Frey to study the effect of biased diffusion in the diffusive part, which is inspired by the fact that molecular motors perform a biased diffusion [28]. It is shown that the phase diagram structure becomes much richer and the density profile in the diffusive part qualitatively changes due to the introduction of biased diffusion.

\section{MODEL}

The sketch of the model is shown in Fig. 1. We study a ring, which separates into two equal-sized parts with size
$N$. In the upper biased diffusive part, a particle could hop either toward the left with rate $D_{1}=q N+\delta$ or hop toward the right with rate $D_{2}=q N-\delta$. As pointed out by Hinsch and Frey [10,21], this scaling can be understood as a time scale separation and ensures competitive behavior between the system's constituents. In the absence of the scaling, the model becomes straightforward to be understood [27]. The lower active part is a TASEP, in which particles hop forward with rate 1 . In the special case $\delta=0$, the model reduces to the one studied in Ref. [21].

\section{RESULTS}

Let us denote particle density $n_{p}=N_{p} /(2 N)$, where $N_{p}$ is the particle number in the ring. Now we carry out analytical investigations of the model. We denote the system flow rate as $J$, and the mean field equation reads

$$
J=D_{1} \rho_{i}\left(1-\rho_{i+1}\right)-D_{2} \rho_{i+1}\left(1-\rho_{i}\right)
$$

in the diffusive part. Taylor expansion over $i$ yields

$$
J=D_{1} \rho_{i}\left(1-\rho_{i}-\frac{d \rho_{i}}{d x} \Delta x\right)-D_{2}\left(\rho_{i}+\frac{d \rho_{i}}{d x} \Delta x\right)\left(1-\rho_{i}\right) .
$$

Substituting $D_{1}=q N+\delta$ and $D_{2}=q N-\delta$ into Eq. (2), omitting the subscript $i$, and replacing $\Delta x$ by $1 / N$, one has

$$
J=2 \delta \rho(1-\rho)-q \frac{d \rho}{d x}+\delta(1-2 \rho) \frac{d \rho}{d x} \frac{1}{N} .
$$

In the limit $N \rightarrow \infty$, the last term on the right hand side could be neglected; thus,

$$
\frac{d \rho}{J-2 \delta \rho(1-\rho)}=-\frac{d x}{q} .
$$

We denote the density of the first and the last sites of the diffusive part as $\rho_{0}$ and $\rho_{1}$, respectively. Integrating Eq. (4), one has

$$
\int_{\rho_{0}}^{\rho_{1}} \frac{d \rho}{J-2 \delta \rho(1-\rho)}=\int_{0}^{1}-\frac{d x}{q} .
$$




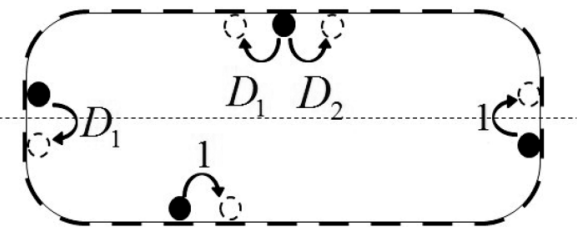

FIG. 1. The sketch of the model.

\section{A. $\delta<0.5$}

We first consider the situation $\delta<0.5$, in which the low density-high density (LD-HD) region separated by a shock could exist.

$L D-H D$ region. As argued in Ref. [21], in the LD-HD region, ${ }^{1} \rho_{1} \rightarrow 0$ in the limit $N \rightarrow \infty$. $\rho_{0}$ is equal to the density in the HD part in the driven part, which means that $\rho_{0}$ is the larger solution of $\rho_{0}\left(1-\rho_{0}\right)=J$.

Now we consider the left hand side of Eq. (5). Two cases should be classified as follows. As shown in the phase diagram (Fig. 2), in the LD-HD region, $\delta$ is always smaller than $2 J$.

Case (i): $0<\delta<2 J$. In this case, Eq. (5) becomes

$$
\left.\left\{\frac{\arctan \left[\frac{\sqrt{\delta}(2 \rho-1)}{\sqrt{2 J-\delta}}\right]}{\sqrt{2 J \delta-\delta^{2}}}\right\}\right|_{\rho_{0}} ^{0}=-\frac{1}{q} \text {. }
$$

Substituting $\rho_{0}=\frac{1+\sqrt{1-4 J}}{2}$ into this equation, $J$ could be solved and $\rho_{0}$ could be obtained accordingly. Thus, the density profile in the diffusive part could be calculated by

$$
\int_{\rho_{0}}^{\rho(x)} \frac{d \rho}{J-2 \delta \rho(1-\rho)}=\int_{0}^{x}-\frac{d x}{q},
$$

which yields

$$
\rho(x)=\frac{1+\sqrt{\frac{2 J-\delta}{\delta}} \tan \left(A-\frac{\sqrt{2 J \delta-\delta^{2}}}{q} x\right)}{2}
$$

with $A=\arctan \left[\frac{\sqrt{\delta}\left(2 \rho_{0}-1\right)}{\sqrt{2 J-\delta}}\right]$. The average density in the biased diffusive part, denoted as $\rho_{d}$, thus could be calculated by

$$
\rho_{d}=\int_{0}^{1} \rho(x) d x=\frac{1}{2}+\frac{q}{2 \delta} \ln \left[\frac{\cos \left(\frac{\sqrt{2 J \delta-\delta^{2}}}{q}-A\right)}{\cos A}\right] \text {. }
$$

Case (ii): $\delta<0$. Equation (5) becomes

$$
\left.\left[\frac{1}{\sqrt{1-2 J / \delta}} \ln \left|\frac{\rho-\frac{1+\sqrt{1-2 J / \delta}}{2}}{\rho-\frac{1-\sqrt{1-2 J / \delta}}{2}}\right|\right]\right|_{\rho_{0}} ^{0}=-\frac{2 \delta}{q} .
$$

Substituting $\rho_{0}=\frac{1+\sqrt{1-4 J}}{2}$ into this equation, $J$ as well as $\rho_{0}$ could be solved. In this case, Eq. (7) yields

$$
\begin{aligned}
\ln \left|\frac{\rho-\frac{1+\sqrt{1-2 J / \delta}}{2}}{\rho-\frac{1-\sqrt{1-2 J / \delta}}{2}}\right|= & \ln \left|\frac{\rho_{0}-\frac{1+\sqrt{1-2 J / \delta}}{2}}{\rho_{0}-\frac{1-\sqrt{1-2 J / \delta}}{2}}\right| \\
& -\frac{2 \delta}{q} x \sqrt{1-2 J / \delta} .
\end{aligned}
$$

\footnotetext{
${ }^{1}$ Note that if we mention the $X$ region (the LD-HD region), it means that the driven part is in phase $X$ (LD-HD). If we mention the $X-Y$ region (the LD-HD-Y region), it means that the driven part is in phase $X$ (LD-HD) and the diffusive part is in phase $Y$.
}

Since $\frac{1+\sqrt{1-2 J / \delta}}{2}>1$ and $\frac{1-\sqrt{1-2 J / \delta}}{2}<0$ when $\delta<0$, Eq. (11) leads to

$$
\rho(x)=\frac{\frac{1+\sqrt{1-2 J / \delta}}{2}+B \frac{1-\sqrt{1-2 J / \delta}}{2} \exp \left(-\frac{2 \delta}{q} x \sqrt{1-2 J / \delta}\right)}{B \exp \left(-\frac{2 \delta}{q} x \sqrt{1-2 J / \delta}\right)+1}
$$

with $B=\frac{-\rho_{0}+\frac{1+\sqrt{1-2 J / \delta}}{2}}{\rho_{0}-\frac{1-\sqrt{1-2 J / \delta}}{2}}$. Thus,

$$
\begin{aligned}
\rho_{d}= & \frac{1}{2}\left(1+\frac{1}{\sqrt{1-2 J / \delta}}\right) \frac{q}{2 \delta} \\
& \times \ln \left[\frac{B+\exp \left(\frac{2 \delta}{q} \sqrt{1-2 J / \delta}\right)}{B+1}\right] \\
& +\frac{1}{2}\left(1-\frac{1}{\sqrt{1-2 J / \delta}}\right) \frac{q}{2 \delta} \\
& \times \ln \left[\frac{B \exp \left(-\frac{2 \delta}{q} \sqrt{1-2 J / \delta}\right)+1}{B+1}\right] .
\end{aligned}
$$

In both cases, the two boundaries of the LD-HD region are determined by $n_{p}=\left(\rho_{d}+\rho_{0}\right) / 2$ and $n_{p}=\left(\rho_{d}+1-\rho_{0}\right) / 2$, respectively.

Maximum current $(M C)$ region. In the $\mathrm{MC}$ region, the current is $J=0.25$. Therefore, when $0<\delta<2 J$ and $\delta<0$, one could obtain $\rho_{d}$ from Eqs. (9) and (13), respectively. Note that $\rho_{d}$ is function of $\rho_{0}$, and $\rho_{0}$ is unknown here. Due to particle conservation, $\rho_{d}=2 n_{p}-0.5$. Thus, $\rho_{0}$ could be solved. Then, one could obtain $\rho_{1}$ via Eq. (5). The boundary between the $\mathrm{MC}$ region and the LD region is determined by $\rho_{0}=0.5$ and the boundary between the $\mathrm{MC}$ region and the HD region is determined by $\rho_{1}=0$.

$H D$ region. In the HD region, the current is $J=\rho_{d r}(1-$ $\left.\rho_{d r}\right)$. Here $\rho_{d r}>0.5$ is the bulk density in the driven part. Moreover, $\rho_{0}$ is also equal to $\rho_{d r}$.

(a) In the two cases $0<\delta<2 J$ and $\delta<0$, one could obtain $\rho(x)$ and accordingly $\rho_{d}$ as before. Note that $\rho_{d}$ is a function of $\rho_{d r}$. Since $\rho_{d}=2 n_{p}-\rho_{d r}, \rho_{d r}$ could be solved.

(b) In the case $\delta>2 J$, as demonstrated below, $\rho(x)$ is always larger than $\frac{1+\sqrt{1-2 J / \delta}}{2}$ in the diffusive part. Thus, the density profile could be obtained,

$\rho(x)=\frac{1+\sqrt{1-2 J / \delta}}{2}\left[\frac{1-C D \exp \left(-\frac{2 \delta}{q} x \sqrt{1-2 J / \delta}\right)}{1-C \exp \left(-\frac{2 \delta}{q} x \sqrt{1-2 J / \delta}\right)}\right]$,

with $C=\frac{\rho_{d r}-\frac{1+\sqrt{1-2 J / \delta}}{2}}{\rho_{d r}-\frac{1-\sqrt{1-2 J / \delta}}{2}}$ and $D=\frac{1-\sqrt{1-2 J / \delta}}{1+\sqrt{1-2 J / \delta}}$. Obviously $\rho(x)$ is always larger than $\frac{1+\sqrt{1-2 J / \delta}}{2}$, because $0<C<1$ and $0<$ $D<1$ (note that $\rho_{d r}=\frac{1+\sqrt{1-4 J}}{2}$ and $\delta<0.5$ ). Thus, $\rho_{d}=$ $\frac{q}{2 \delta} \ln \left[\frac{C-\exp (E)}{C-1}\right]-\frac{\sqrt{1-2 J / \delta}}{2}+\frac{1}{2}$, with $E=\frac{2 \delta}{q} \sqrt{1-2 J / \delta}$.

(c) In the case $\delta=2 J$, the integration

$$
\int \frac{d \rho}{J-2 \delta \rho(1-\rho)}=\frac{1}{2 \delta} \int \frac{d \rho}{(\rho-1 / 2)^{2}}=-\frac{1}{2 \delta(\rho-1 / 2)} \text {. }
$$

As demonstrated below, $\rho(x)$ is always larger than $1 / 2$ in the diffusive part. Therefore, the density profile could be obtained 

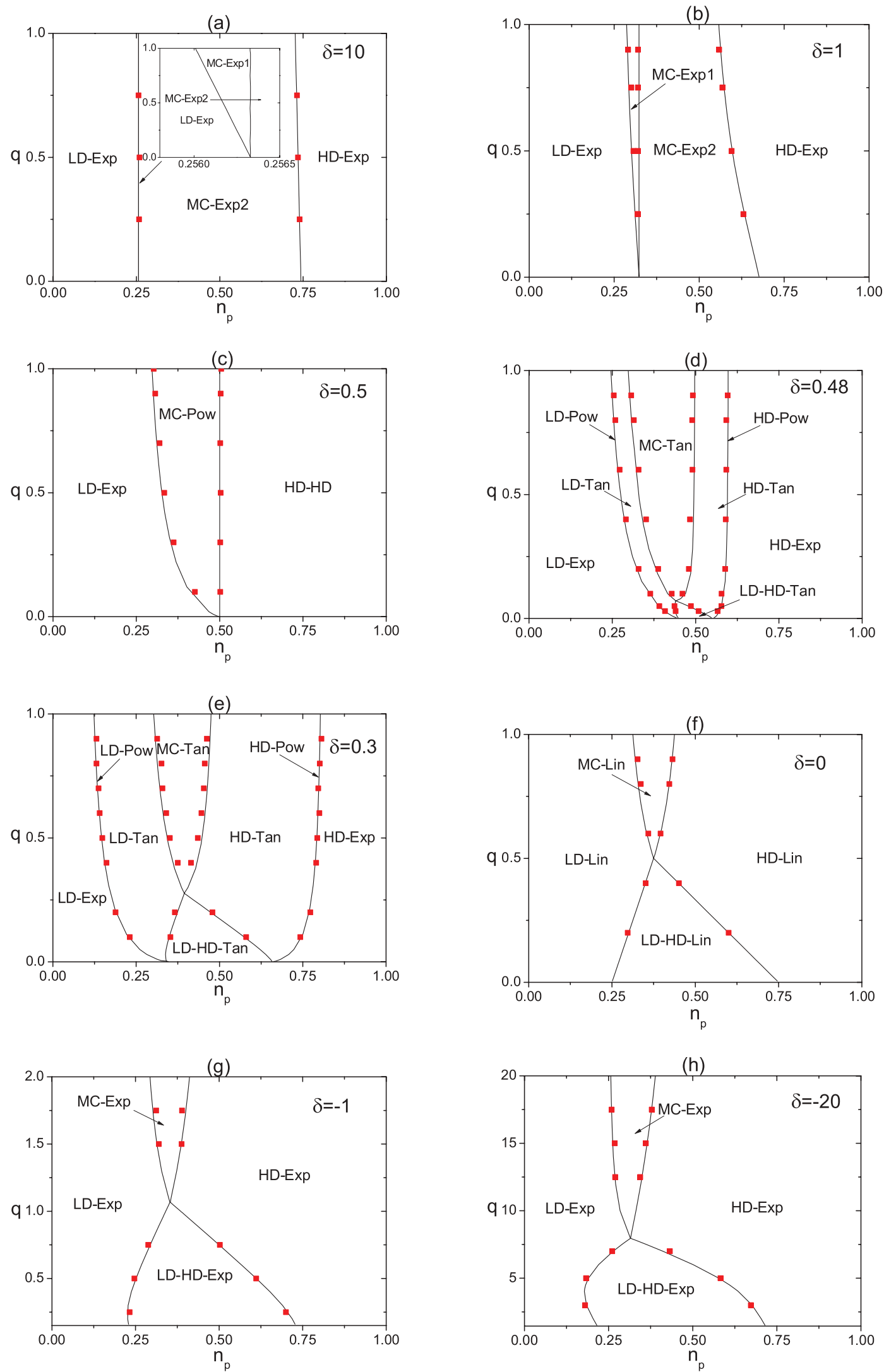

FIG. 2. (Color online) Analytical results (black lines) and Monte Carlo simulation results (red squares) of phase diagram at different values of $\delta$. From (a) to (h), $\delta=10,1,0.5,0.48,0.3,0,-1,-20$. In (d) and (e), lines LD-Pow and HD-Pow correspond to $\delta=2 J$. Between the two lines, $\delta<2 J$, and beyond the two lines, $\delta>2 J$. The inset in (a)shows the details for the MC-Exp1 phase. Note that the MC-Exp1 phase is so narrow that it cannot be distinguished from the simulations. Simulation size is $N=500$. 
as follows:

$$
\rho(x)=\frac{1}{\frac{2 \delta}{q} x+\frac{1}{\rho_{d r}-1 / 2}}+\frac{1}{2} .
$$

Obviously $\rho(x)$ is always larger than $1 / 2$. Thus, $\rho_{d}=\frac{q}{2 \delta}$ $\ln \left[\frac{2 \delta}{q}\left(\rho_{d r}-\frac{1}{2}\right)+1\right]+\frac{1}{2}$. The curve of $\delta=2 J$ is determined by

$$
\rho_{d}+\rho_{d r}=2 n_{p} .
$$

$L D$ region. In the $\mathrm{LD}$ region, the current is still $J=$ $\rho_{d r}\left(1-\rho_{d r}\right)$. Here $\rho_{d r}<0.5$. Since $\rho_{1}=0$ in the LD region, the density profile in the diffusive part could be obtained:

$$
\int_{\rho(x)}^{0} \frac{d \rho}{J-2 \delta \rho(1-\rho)}=\int_{x}^{1}-\frac{d x}{q} .
$$

(a) In the two cases $0<\delta<2 J$ and $\delta<0$, together with $\rho_{d}=2 n_{p}-\rho_{d r}, \rho_{d r}$ could be solved.

(b) In the case $\delta>2 J, \rho(x)$ is always smaller than $\frac{1-\sqrt{1-2 J / \delta}}{2}$ in the diffusive part. Therefore, the density profile could be obtained:

$\rho(x)=\frac{1-\sqrt{1-2 J / \delta}}{2}\left\{\frac{\exp \left[\frac{2 \delta}{q}(1-x) \sqrt{1-2 J / \delta}\right]-1}{\exp \left[\frac{2 \delta}{q}(1-x) \sqrt{1-2 J / \delta}\right]-D}\right\}$.

Thus,

$\rho_{d}=\frac{1-\sqrt{1-2 J / \delta}}{2}\left\{1+\frac{(1-D) \ln \left[\frac{(1-D) \exp (E)}{\exp (E)-D}\right]}{D E}\right\}$.

(c) In the case $\delta=2 J, \rho(x)$ is always smaller than $1 / 2$ in the diffusive part. Therefore, the density profile is

$$
\rho(x)=\frac{1}{2}\left[1-\frac{1}{\frac{\delta}{q}(1-x)+1}\right]
$$

and $\rho_{d}=\frac{1}{2}-\frac{q}{2 \delta} \ln \left(1+\frac{\delta}{q}\right)$. The curve of $\delta=2 J$ in the LD region is still determined by Eq. (17).

Figures 2(d)-2(h) show the phase diagram at different values of $\delta<0.5$. When $0<\delta<0.5$, there are eight phases [Figs. 2(d) and 2(e)]. Note that here $X$-Pow corresponds to the boundary separating $X$-Exp and $X$-Tan. Here "Tan" ("Exp", "Pow") means that the density profile in the diffusive part follows a tangent-related (an exponential-related, a powerlaw-related) function. Phases LD-Exp and HD-Exp shrink, and phases $X$-Tan expand with the decrease of $\delta$. When $\delta=0$, phases $X$-Tan transit into phase $X$-Lin [Fig. 2(f)] and other phases disappear. Here "Lin" means linear density profile in the diffusive part. When $\delta$ becomes negative, phases $X$-Lin transit into phases $X$-Exp. With the further decrease of $\delta$, phase LD-HD-Exp expands and the other three phases shrink [Figs. 2(g) and 2(h)].

\section{B. $\delta=0.5$}

In the range $0<\delta<0.5$, phases LD-HD-Tan, LD-Tan, and HD-Tan shrink with the increase of $\delta$. The boundary between MC-Tan and HD-Tan becomes more and more straight [Figs. 2(d) and 2(e)]. When $\delta=0.5$, the three phases
LD-HD-Tan, LD-Tan, and HD-Tan disappear, the phase LDExp remains, the phase MC-Tan transits into MC-Pow, and the phase HD-Exp transits into HD-HD [Fig. 2(c)].

Now we analyze the three phases MC-Pow, LD-Exp, and HD-HD. In the phase MC-Pow, with $J=0.25$ and $\delta=0.5$, the density profile in the diffusive part is

$$
\int_{\rho_{0}}^{\rho(x)} \frac{d \rho}{0.25-\rho(1-\rho)}=\int_{0}^{x}-\frac{d x}{q},
$$

which leads to

$$
\rho(x)=0.5+\frac{\left(\rho_{0}-0.5\right) q}{\left(\rho_{0}-0.5\right) x+q}
$$

since $\rho(x)$ is always smaller than $1 / 2$ in the phase. Thus,

$$
\rho_{d}=0.5+q \ln \left(\frac{\rho_{0}-0.5+q}{q} .\right)
$$

Together with $\rho_{d}+0.5=2 n_{p}, \rho_{0}$ as well as $\rho_{1}$ could be solved. The two boundaries of the phase are determined by $\rho_{1}=0$ and $\rho_{0} \rightarrow 0.5$. Note that $\rho=0.5$ is a divergence point in Eq. (22). When $\rho_{0}=0.5$, one knows that the density profile should be flat $[\rho(x)=0.5]$ from Eq. (3), which corresponds to $n_{p}=0.5$ [see, e.g., $\delta=0.5$ in Fig. 3 (a) and $n_{p}=0.5$ in Fig. 4(b)].

In the HD-HD phase, with $\delta=0.5$, the density profile in the diffusive part is

$$
\int_{\rho_{d r}}^{\rho(x)} \frac{d \rho}{J-\rho(1-\rho)}=\int_{0}^{x}-\frac{d x}{q} .
$$

However, since $\rho_{d r}=\frac{1+\sqrt{1-4 J}}{2}$ is the divergence point in this equation, Eq. (25) is invalid. In this case, one knows that the density profile should be flat $\left[\rho(x)=\frac{1+\sqrt{1-4 J}}{2}\right]$ from Eq. (3), which is equal to the bulk density in the drive part [see, e.g., $n_{p}=0.6$ in Fig. 4(b)].

Finally, in the LD-Exp phase, with $\delta=0.5$, the density profile in the diffusive part is

$$
\int_{\rho(x)}^{0} \frac{d \rho}{J-\rho(1-\rho)}=\int_{x}^{1}-\frac{d x}{q},
$$

which leads to

$$
\rho(x)=\frac{1-\sqrt{1-4 J}}{2}\left\{\frac{\exp [(1-x) E]-1}{\exp [(1-x) E]-D}\right\}
$$

since $\rho(x)$ is always smaller than $\frac{1-\sqrt{1-4 J}}{2}$ in the phase.

$$
\text { C. } \delta>0.5
$$

When $\delta>0.5$, four phases exist in the phase diagram as can be seen from Figs. 2(a) and 2(b). In particular, there are two MC regions. In the MC-Exp1 (MC-Exp2) phase, $\rho(x)$ is monotonically decreasing (increasing) with $x$ in the diffusive part. With the increase of $\delta$, phase MC-Exp2 expands and phase MC-Exp1 shrinks. 

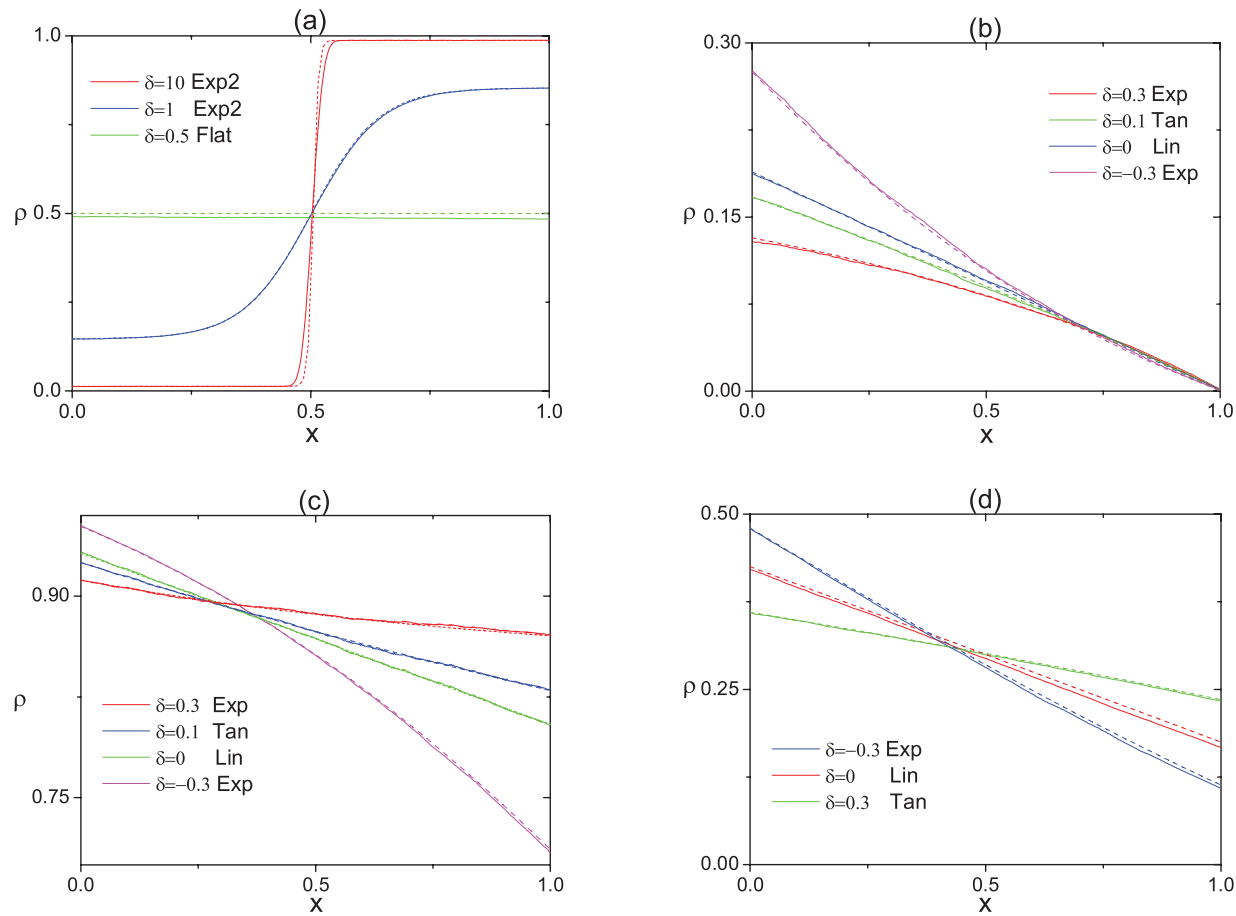

FIG. 3. (Color online) Typical density profiles: (a-d) the diffusive part, and the corresponding driven part is in MC, LD, HD, and MC, respectively (not shown); (e, g) the diffusive part; and (f, h) the corresponding driven part. The solid lines are simulation results and the dashed lines are analytical ones. The parameters are (a) $n_{p}=0.5, q=$ 0.1 ; (b) $n_{p}=0.1, q=0.5$; (c) $n_{p}=$ $0.9, q=0.5$; (d) $n_{p}=0.4, q=1.0$; (e, f) $n_{p}=0.5, q=0.1 ;(\mathrm{g}, \mathrm{h}) \delta=$ $-20, n_{p}=0.5$. Simulation size is $N=500$.
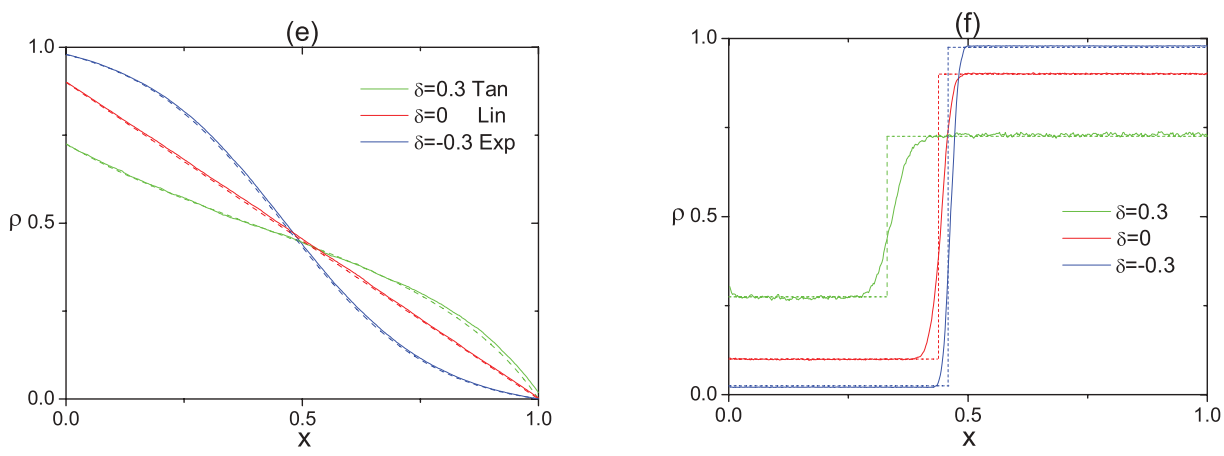

(g)
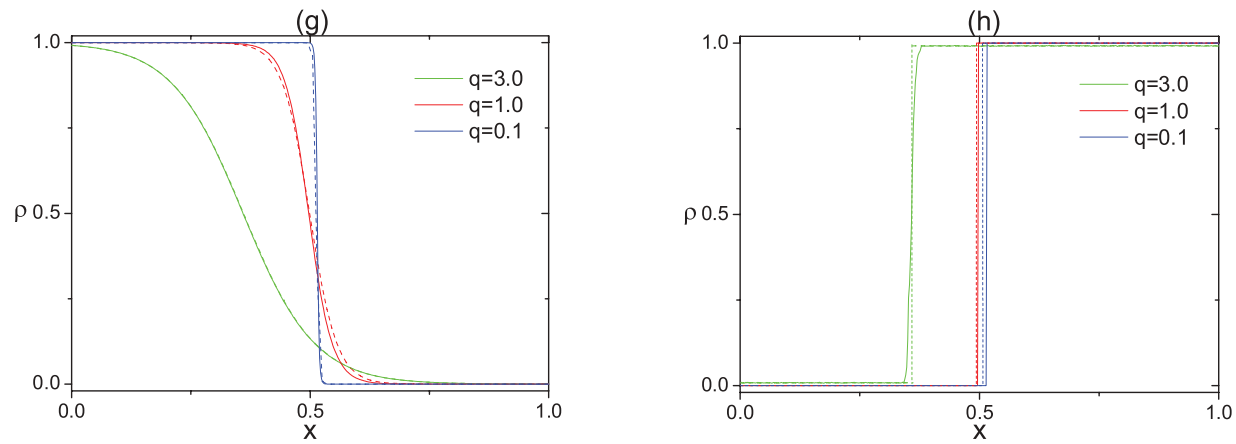

In the two phases, with $J=0.25$, the density profile in the diffusive part is

$$
\int_{\rho_{0}}^{\rho(x)} \frac{d \rho}{0.25-2 \delta \rho(1-\rho)}=\int_{0}^{x}-\frac{d x}{q} .
$$

In the MC-Exp1 phase, $\rho(x)$ is always smaller than $\frac{1-\sqrt{1-1 / 2 \delta}}{2}$; thus,

$$
\rho(x)=\frac{1}{2}-\frac{F}{1+\frac{2}{\left[\frac{F+\left(0.5-\rho_{0}\right)}{F-\left(0.5-\rho_{0}\right)} \exp \left(-\frac{4 \delta F x}{q}\right)\right]-1}},
$$

with $F=\frac{\sqrt{1-1 / 2 \delta}}{2}$, is monotonically decreasing.

In the MC-Exp2 phase, $\rho(x)$ is always larger than $\frac{1-\sqrt{1-1 / 2 \delta}}{2}$ and smaller than $\frac{1+\sqrt{1-1 / 2 \delta}}{2}$; thus,

$$
\rho(x)=\frac{1}{2}-\frac{F}{1+\frac{2}{\left[\frac{F+\left(0.5-\rho_{0}\right)}{F-\left(0.5-\rho_{0}\right)} \exp \left(-\frac{4 \delta F x}{q}\right)\right]-1}}
$$

is monotonically increasing.

On the boundary separating the two MC phases, the density profile is flat $\left[\rho(x)=\frac{1-\sqrt{1-1 / 2 \delta}}{2}\right]$ in the diffusive part [see, e.g., $n_{p}=0.33$ in Fig. 4(a)]. 
(a)

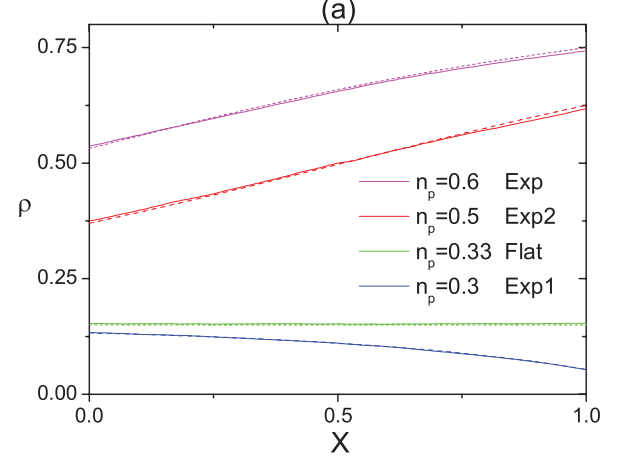

(b)

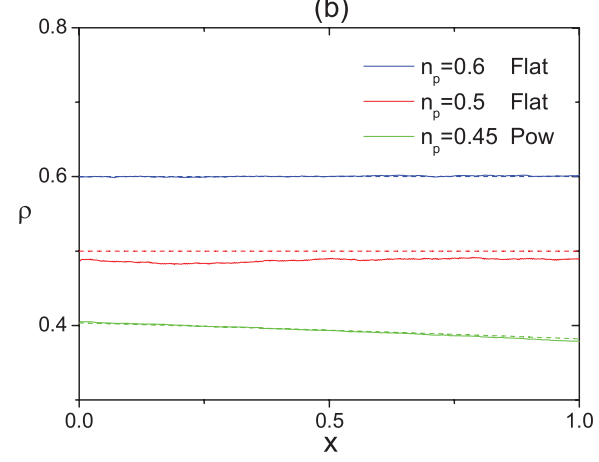

(c)

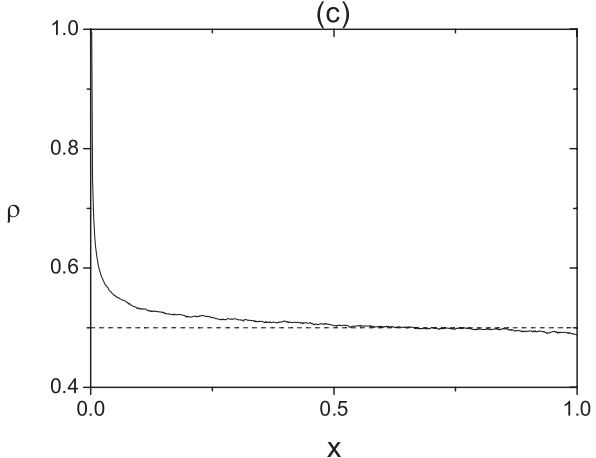

FIG. 4. (Color online) Typical density profiles: $(\mathrm{a}, \mathrm{b})$ the diffusive part. (a) The corresponding driven part is in HD for $n_{p}=0.6$ and in MC for $n_{p}=0.5,0.33$, and 0.3 (not shown). (b) The corresponding driven part is in HD for $n_{p}=0.6$ and in MC for $n_{p}=0.45$ (not shown). (c) The corresponding driven part is in $\mathrm{MC}$ for $n_{p}=0.5$. The solid lines are simulation results and the dashed lines are analytical ones. The parameters are (a) $\delta=1, q=0.9$; (b, c) $\delta=0.5, q=0.5$. Simulation size is $N=500$.

In the HD-Exp phase, $\rho(x)$ is always smaller than $\frac{1+\sqrt{1-2 J / \delta}}{2}$ and larger than $\frac{1-\sqrt{1-2 J / \delta}}{2}$, and it is also determined by Eq. (14). However, since $C<0$, the density profile is monotonically increasing.

In the LD-Exp phase, $\rho(x)$ is always smaller than $\frac{1-\sqrt{1-2 J / \delta}}{2}$, and it is also determined by Eq. (19).

\section{Simulation results}

Figures 3 and 4 show the typical density profiles; the analytical results are in good agreement with simulation ones. We would like to point out that when both $\delta$ and $n_{p}$ are very close to 0.5 , the simulation results slightly deviate from the analytical ones; see, e.g., the density profile of $n_{p}=0.5$ in Fig. 4(b). This is because the system size is finite. As a result, the driven part has a non-negligible boundary layer [see, e.g., the left boundary layer in Fig. 4(c), which extends into the bulk] and thus its average density slightly deviates from the analytical prediction. Consequently, the density profile of the diffusive part slightly deviates from the analytical result due to particle conservation. One expects that with the increase of system size, the boundary layer is gradually restricted in the very vicinity of the boundaries; thus, the deviation will decrease and disappear asymptotically. However, as pointed out in Ref. [22], correct Monte Carlo results may not be retrieved in the large-size limit if the random-number generator produces a pseudorandom number series (pseudo-RNS) instead of a true RNS.
When $\delta<0,|\delta|$ is large and $q$ is small [see Fig. 3(g)], the flow rate in the system approaches zero, and the density profile in the diffusive part forms an antishock. The densities upstream and downstream of the antishock approximately equal 1 and 0 , respectively. However, the densities upstream and downstream of the shock in the driven part approximately equal 0 and 1 , respectively [Fig. 3(h)].

On the other hand, when $\delta>0$ and $|\delta|$ is large, the flow rate in the system also approaches zero, and the density profile in the diffusive part forms a shock in the MC-Exp2 phase [Fig. 3(a)]. The densities upstream and downstream of the shock approximately equal 0 and 1 , respectively. As a result, the boundaries separating the LD and HD phase from the MCExp2 phase gradually approach the straight line $n_{p}=0.25$ and $n_{p}=0.75$, respectively [Fig. 2(a)]. Note that the MC-Exp1 phase is extremely small when $\delta$ is large.

The difference in density profile in the diffusive part is because the domain wall is behaving differently. The domain wall is the object that separates bulk domains of different dynamic phases. It has been shown that properties of asymmetric exclusion processes can be well described and understood via random-walk dynamics of the domain wall (see Refs. [6,29]). If the motion of the domain wall is biased then one of the corresponding dynamic phases wins and it occupies the whole system in the stationary-state limit. The phase boundary corresponds to the situation when the motion of the domain wall is unbiased. If the diffusion rate for the domain in this case is independent of the position at large times then a linear particle density profile is observed. However, in 
our system the entrance and exit rates to different parts of the lattice are not independent, and it leads to position-dependent diffusion of the domain wall. The domain wall moves in an effective potential created by this coupling. When the potential is flat, the domain wall diffuses equally in each direction and we have a linear profile. All other cases (Exp, Tan, and Pow) are the result of the bias in the motion of the domain wall.

\section{CONCLUSION}

To summarize, this paper has investigated the effect of biased diffusion in a mesoscopic ring composed of a driven part and a diffusive part. The dependence of the phase diagram and density profile on the biased diffusion parameter $\delta$ have been studied. Analytical investigations have shown that the density profile in the diffusive part could follow various functions (exponential, tangent, power-law, or linear, because the domain wall is behaving differently), be flat, or be in a shock or antishock. Consequently, rich phase diagram structures have been observed. Our analytical results are in good agreement with Monte Carlo simulations.

\section{ACKNOWLEDGMENTS}

We are thankful for the comments of two anonymous referees, which enabled us to find that our analytical results for $\delta \geqslant 0.5$ were incorrect in the first version of this paper. This work is supported by the National Basic Research Program of China (Grant No. 2012CB725404), the NNSFC (Grants No. 11072239 and No. 71171185), and the Fundamental Research Funds for the Central Universities (Grant No. WK2320000014).
[1] B. Schmittmann and R. K. P. Zia, Phys. Rep. 301, 45 (1998).

[2] B. Derrida, Phys. Rep. 301, 65 (1998).

[3] R. A. Blythe and M. R. Evans, J. Phys. A 40, R333 (2007).

[4] T. Chou, K. Mallick, and R. K. P. Zia, Rep. Prog. Phys. 74, 116601 (2011).

[5] D. Chowdhury, L. Santen, and A. Schadschneider, Phys. Rep. 329, 199 (2000).

[6] G. Schütz, in Phase Transitions and Critical Phenomena, edited by C. Domb and J. Lebowitz (Academic Press, London, 2000).

[7] B. Schmittmann and R. Zia, in Phase Transitions and Critical Phenomena, edited by C. Domb and J. Lebowitz (Academic Press, London, 1995).

[8] S. Katz, J. L. Lebowitz, and H. Spohn, J. Stat. Phys. 34, 497 (1984).

[9] M. R. Evans, D. P. Foster, C. Godreche, and D. Mukamel, Phys. Rev. Lett. 74, 208 (1995); M. R. Evans, Y. Kafri, H. M. Koduvely, and D. Mukamel, ibid. 80, 425 (1998).

[10] A. Parmeggiani, T. Franosch, and E. Frey, Phys. Rev. Lett. 90, 086601 (2003).

[11] S. A. Janowsky and J. L. Lebowitz, Phys. Rev. A 45, 618 (1992); J. Stat. Phys. 77, 35 (1994).

[12] A. B. Kolomeisky, J. Phys. A 31, 1153 (1998).

[13] B. Derrida, M. R. Evans, and V. Hakim, J. Phys. A 26, 1493 (1993); B. Derrida, E. Domany, and D. Mukamel, J. Stat. Phys. 69, 667 (1992).
[14] G. Schütz and E. Domany, J. Stat. Phys. 72, 277 (1993).

[15] J. Krug, Phys. Rev. Lett. 67, 1882 (1991).

[16] V. Popkov and G. M. Schütz, Europhys. Lett. 48, 257 (1999).

[17] V. Popkov, L. Santen, and A. Schadschneider, J. Phys. A 34, L45 (2001).

[18] C. Appert and L. Santen, Phys. Rev. Lett. 86, 2498 (2001).

[19] D. Helbing, Rev. Mod. Phys. 73, 1067 (2001).

[20] F. Peruani, T. Klauss, A. Deutsch, and A. Voss-Boehme, Phys. Rev. Lett. 106, 128101 (2011).

[21] H. Hinsch and E. Frey, Phys. Rev. Lett. 97, 095701 (2006).

[22] R. Jiang, W. Huang, M. B. Hu, Y. M. Yuan, and Q. S. Wu, Phys. Rev. Lett. 106, 079601 (2011).

[23] B. Derrida, J. L. Lebowitz, and E. R. Speer, J. Stat. Phys. 107, 599 (2002).

[24] K. Jain, R. Marathe, A. Chaudhuri, and A. Dhar, Phys. Rev. Lett. 99, 190601 (2007).

[25] K. Tsekouras and A. B. Kolomeisky, J. Phys. A 41, 465001 (2008).

[26] J. E. Santos and G. M. Schütz, Phys. Rev. E 64, 036107 (2001).

[27] Q. H. Shi, R. Jiang, M. B. Hu, and Q. S. Wu, Phys. Rev. E 85, 041131 (2012).

[28] C. Bustamante, D. Keller, and G. Oster, Acc. Chem. Res. 34, $412(2001)$

[29] A. B. Kolomeisky et al., J. Phys. A: Math. Gen. 31, 6911 (1998). 